Macedonian Pharmaceutical Bulletin, 66 (Suppl 1) 215 - 216 (2020)

Online ISSN $1857-8969$

UDC: 615.214.32:[582.684.1:547.56

DOI: 10.33320/maced.pharm.bull.2020.66.03.107

Short communication

\title{
Molecular docking of monoamine oxidase A with xanthones from Hypericum perforatum roots
}

\author{
Marija Todorovska*, Jovana Georgieva, Oliver Tusevski, Sonja Gadzovska Simic \\ Laboratory of Plant Cell and Tissue Culture, Institute of Biology, Faculty of Natural Sciences and Mathematics, \\ Ss. Cyril and Methodius University, Arhimedova 3, 1000 Skopje, Republic of North Macedonia
}

\section{Introduction}

Hypericum perforatum L. (St John's wort) represents the most studied medicinal plant throughout the world due to the presence of a broad range of secondary metabolites with biological activities. Phenolic compounds, such as flavonoids naphtodianthrones, phloroglucinols and xanthones are the main bioactive metabolites commonly described for this plant (Nahrstedt and Butterweck, 2010). The most significant use of $H$. perforatum preparations comprises symptomatic treatments of mild-to-moderate depression and recently good perspectives emerged for major depression (Solomon et al., 2013). The in vitro studies for biological activity of various compounds from $H$. perforatum extracts showed the monoamine oxidaseA (MAO-A) inhibition as the possible mechanism for antidepressant effect (Thiede and Walper, 1994).

The antidepressant activity of $H$. perforatum has been related to the hypericins, hyperforins and flavonoids accumulated in the aerial plant parts. Recently, root extracts of $H$. perforatum have been recognized as the main source of xanthones with MAO-A inhibitory properties highlighting their in vitro antidepressant effects (Tusevski et al., 2018). However, the action mechanism of xanthones from H. perforatum roots for MAO-A inhibition has never been examined. In this study, molecular docking analysis was employed for the first time to elucidate molecular interactions between MAO-A with xanthones as the most abundant metabolites from $H$. perforatum roots.

\section{Materials and methods}

Enzyme preparation

The crystallographic structure of MAO-A enzyme (pdb: 2Z5X) in complex with harmine was downloaded from the Protein Data Bank RSCB PDB. The raw crystal structure of MAO-A was prepared with AutoDock Tools 4.2 where all water molecules, ligands and co-factors were removed, while Kollman united-atom partial charges for neutralization of the enzyme were added, as well non-polar hydrogens were merged. All hydrogen atoms of MAO-A were further optimized by using the MolProbity application to generate a correct hydrogen bond network. This enzyme structure was saved in pdbqt format in AutoDock tools 4.2.

\section{Ligand preparation}

Mangiferin and $\gamma$-mangostin were selected as the most representative ligands for molecular docking study due to their abundance in $H$. perforatum roots (Tusevski et al., 2018). The ligand molecules were downloaded from PubChem database. Atomic charge and potential of the ligands were computed with VEGA ZZ program (3.1.2) using TRIPOS force field along with Gasteiger charges. The prepared

\footnotetext{
* todorovskabt96@gmail.com
} 
ligand structures were then saved in pdbqt format in AutoDock tools 4.2.

\section{Molecular docking}

AutoDock 4.2 software package was used to predict the molecular interactions between the representative ligands and the MAO-A receptor by using the Lamarckian Genetic Algorithm. Standard docking protocol for rigid protein and flexible ligands was implemented with 10 independent runs per ligand. AutoGrid 4.2 program was used to calculate grid maps of $60 \times 60 \times 60$ with $0.375 \AA$ distance between grid points. The best ligand binding conformation was selected according to the lowest binding energy $\left(\mathrm{kcal} \cdot \mathrm{mol}^{-1}\right)$, as well the type of interaction between the ligand atoms and enzyme amino acid residues. The best docking results were analyzed and visualized using the Discovery Studio Visualizer 16.1 (Accelrys, San Diego, CA, USA).

\section{Results and Discussion}

The docking data for MAO-A showed that $\gamma$ mangostin and mangiferin exhibited different binding energies towards the active site of the enzyme. Among two tested xanthones, $\gamma$-mangostin showed the most favourable interaction into MAO-A pocket that was represented with the lowest binding energy $\left(-9.97 \mathrm{kcal} \cdot \mathrm{mol}^{-1}\right)$. In this context, $\gamma$ mangostin was found as the most prominent MAOA inhibitor due to the formation of hydrogen bonds of $\mathrm{OH}$ groups at $\mathrm{C} 3$ and $\mathrm{C} 6$ positions from phenyl rings $\mathrm{C}$ and $\mathrm{A}$ with amino acids Phe 208 and Asn 181, respectively. In accordance with this finding, the structure-activity relationship of oxygenated xanthones highlighted the importance of $\mathrm{OH}$ groups at $\mathrm{C} 3$ and $\mathrm{C} 6$ position for effective MAO-A inhibitory activity (Ji and Zhang, 2006). Additionally, $\gamma$-mangostin-enzyme complex was stabilized through hydrophobic interactions with amino acid residues Phe $208(\pi-\pi$ T-shaped), Tyr 407, Tyr $444(\pi-\pi$ stacked) and FAD cofactor $(\pi-$ sigma). Our docking results showed that the planar rings of $\gamma$-mangostin along with their prenyl substituents at $\mathrm{C} 2$ and $\mathrm{C} 8$ positions are the main structures responsible for hydrophobic interactions into the MAO-A pocket.

Mangiferin as the representative of glycosylated xanthones exhibited moderate inhibition towards MAO-A with binding energy of $-5.16 \mathrm{kcal} \cdot \mathrm{mol}^{-1}$. In the study of Gnerre et al. (2001), mangiferin was shown less active in MAO-A inhibition than its aglycone form due to a glucose moiety that interrupt its fitting into the enzyme active site. Docking pose of mangiferin into MAO-A active site was stabilized by the formation of numerous hydrogen bonds with amino acid residues Gly 443, Phe 208, Cys 323, Asn 181, Thr 336 and Tyr 407 and several hydrophobic interactions with Ile 180 ( $\pi$-alkyl), Tyr 407 and Tyr $444(\pi-\pi$ stacked), as well the cofactor FAD $(\pi-\pi$ Tshaped). Even that mangiferin is weaker MAO-A inhibitor, its high concentration in $H$. perforatum root extracts could additionally contributed to the antidepressant effects.

\section{Conclusion}

Computational approach performed in the present study highlighted the action mechanism of xanthones from $H$. perforatum roots for MAO-A inhibition. Molecular docking data revealed that $\gamma$ mangostin and mangiferin are promising antidepressant compounds due to their capacity for establishment of hydrogen binding and hydrophobic interactions with MAO-A active site. This study provides pivotal evidence for selecting xanthones from $H$. perforatum roots as potential compounds for prevention and treatment of depression.

\section{References}

Gnerre, C., Thull, U., Gaillard, P., Carrupt, P.A., Testa, B., Hostettmann, K., 2001. Natural and synthetic xanthones as monoamine oxidase inhibitors: biological assay and 3D-QSAR. Helv. Chim. Acta 84, 552-570.

Ji, H.F., Zhang, H.Y., 2006. Theoretical evaluation of flavonoids as multipotent agents to combat Alzheimer's disease. J. Mol. Struc-THEOCHEM 767, 3-9.

Nahrstedt, A., Butterweck, V., 2010. Lessons Learned from Herbal Medicinal Products: The Example of St. John's Wort. J. Nat. Prod. 73, 1015-1021.

Solomon, D., Adams, J., Graves, N., 2013. Economic evaluation of St. John's wort (Hypericum perforatum) for the treatment of mild to moderate depression. J. Affect. Disord. 148, 228-234.

Thiede, H.M., Walper, A., 1994. Inhibition of MAO and COMT by Hypericum extracts and hypericin. J. Geriatr. Psych. Neur. 7, S54-S56.

Tusevski, O., Krstikj, M., Stanoeva, J.P., Stefova, M., Gadzovska Simic, S., 2018. Phenolic profile and biological activity of Hypericum perforatum L.: Can roots be considered as a new source of natural compounds. S. Afr. J. Bot. 117, 301-310.

Maced. Pharm. Bull. 66 (Suppl 1) 215 - 216 (2020) 